\title{
Recurrent and unusual manifestations of tuberculosis in a boy with interleukin 12 receptor defect
}

M P Senanayake ${ }^{1}$, R Doffinger ${ }^{2}$, D S Kumararatne ${ }^{2}$

\section{Introduction}

A genetically determined susceptibility to mycobacterial disease due to a specific immunological deficiency has been described [1,2]. Although known as Mendelian susceptibility to mycobacterial disease (MSMD) this entity has both clinical and genetic heterogeneity. We describe a Sri Lankan boy with recurrent and rare manifestations of mycobacterial disease in whom a complete interleukin 12 receptor defect was identified.

\section{Case report}

An emaciated and ill looking 13-year old boy of non consanguinous Sri Lankan parentage presented with a swollen painful right knee of a few days duration. He had cough, fever and multiple large non-healing skin ulcers of limbs and trunk (Figure 1). The skin lesions had begun as subcutaneous nodules that progressed and were resistant to surgical drainage and antibiotics over a period of nine months. There were bilateral, non-tender cervical lymph nodes and an enlarged liver.

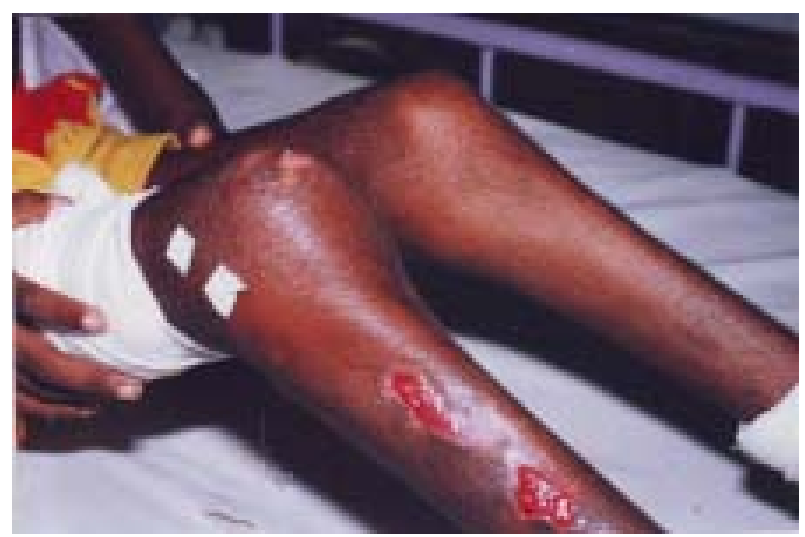

Figure 1. Large non-healing skin ulcers and swollen right knee.

In infancy he had developed biopsy proven tuberculous cervical lymphadentitis within six months of vaccination with BCG and was treated in the local hospital.
A prominent scar was visible but he had tested tuberculin negative. No other infections were reported until eight years when a diagnosis of tuberculous pleural effusion was made. He was again tuberculin negative but responded to isoniazid, rifampicin and ethambutol. He lived with his mother who was healthy. All contact was lost with the father whose health status was not known.

Biopsy from edge of ulcers showed epidermal hyperplasia with diffuse inflammatory cell infiltrate of macrophages, plasma cells, neutrophils and Langerhan type multinucleated giant cells suggesting tuberculous aetiology. A positive DNA amplification polymerase chain reaction specific for Mycobacterium tuberculosis was found on joint aspirate. Treatment with isoniazid, rifampicin, pyrazinamide and ethambutol resulted in complete resolution of skin ulcers, lung infection and joint swelling (Figure 2).

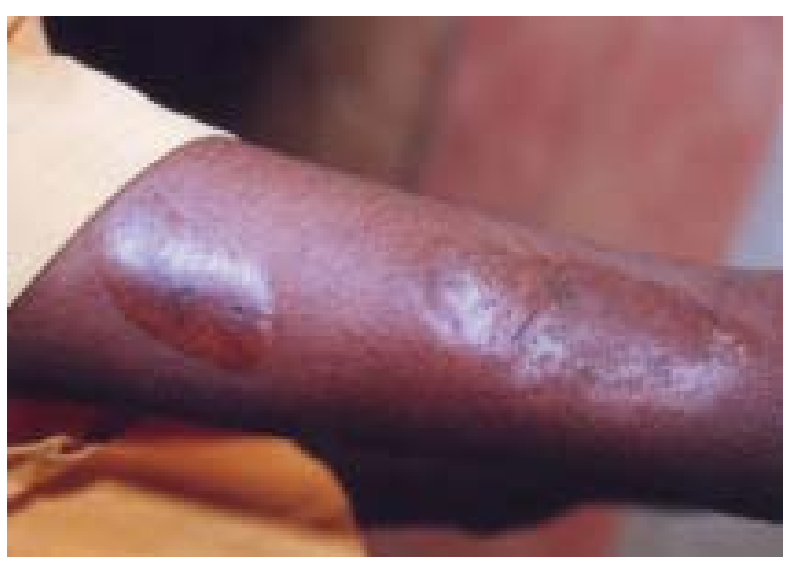

Figure 2. Healed ulcers with scar tissue formation following antituberculosis therapy.

Investigations for immune deficiency were undertaken due to recurrent unusual manifestations of tuberculosis with persistent tuberculin anergy. Serum immunoglobulin levels, CD 4 and CD 8 levels, T-cell and B-cell counts and nitro blue tetrazolium test were normal and HIV virus was negative. Tests done on patient's blood and a healthy travel sample in the UK, showed evidence of interleukin 12 receptor deficiency (Table 1).

${ }^{1}$ Department of Paediatrics, University of Colombo, Sri Lanka and ${ }^{2}$ Department of Immunology, Addenbrookes Hospital, Cambridge, UK.

Correspondence: MPS, e-mail <manouri.senanayake@gmail.com>. Received on 21 August 2008 and accepted on 14 February 2009. Competing interests: none declared 
Table 1. IFN gamma production (pg/ml) - (whole blood stimulation)

\begin{tabular}{lrr}
\hline Stimulus & Control & Patient \\
\hline Medium & 0.0 & 0.0 \\
IL12 & 216.0 & 0.0 \\
PHA & 1419.0 & 404.8 \\
PHA+IL12 & $>5000.0$ & 570.0 \\
IL1b & 11.7 & 2.5 \\
IL12+IL1b & 103.0 & 1.5 \\
IL18 & 6.0 & 2.5 \\
IL12+IL18 & $>5000.0$ & 70.0 \\
LPS & 15.0 & 9.0 \\
LPS/IL12 & 1161.5 & 13.6 \\
BCG & 5.0 & 0.0 \\
BCG/IL12 & 452.0 & 0.0 \\
PMA/IONO & $>5000.0$ & $>5000.0$ \\
\hline
\end{tabular}

\section{Discussion}

Mendelian susceptibility to mycobacterial disease is described and should be considered in children in whom recurrent or unusual forms of tuberculosis or opportunistic mycobacteriosis are encountered. Unlike in classic immunodeficiency the patients rarely present with other infections. Although autosomal recessive and X-linked recessive patterns of inheritance have been described sporadic cases are known. Disseminated infection following live BCG vaccination is described in 25 of 34 such children.

Our patient developed a rare manifestation of cutaneous tuberculosis with subsequent dissemination suggesting the diagnosis of miliary cutaneous tuberculosis which is an entity seen mainly in children. Although atypical mycobacteria is commoner in these patients we found mycobacterial tuberculosis to be the causative species. Immunological studies found significantly reduced IFN gamma production and absent response to IL12. This excludes classical defects in the IFN gamma pathway including IFN gamma receptor 1 and 2 and STAT1. Strong production of TNF and IL6 argue against known defects in the $\mathrm{Nfkb} / \mathrm{Nemo}$ axis. The results were compatible with complete deficiency of IL12R or of a pathway component [3]. Response to treatment is good in these patients but life long prophylaxis is recommended and our patient was placed on isoniazid.

\section{References}

1. Casanova JL, Abel L. Genetic dissection of immunity to mycobacteria: the human model. Annual Review of Immunology 2002; 20: 581-620.

2. Casanova JL, Blanch S, Emilie JF, Jouanguy E, Lamhamedi $\mathrm{S}$, et al. Idiopathic disseminated Bacillus Calmette-Guerin infection: a French national retrospective study. Paediatrics 1996; 98 : 774-8.

3. Altare F, Durandy A, Lammas D, Emile JF, Lamhamedi S, et al. Impairment of mycobacterial immunity in human interleukin 12 receptor-deficient patients. Science 1998; 280: 1435-8. 